\title{
Kenaf (Hibiscus cannabinus L.) seed and its potential food applications: a review
}

\begin{abstract}
Kenaf belongs to the family Malvaceae noted for their economic and horticultural importance. Kenaf seed is a valuable component of kenaf plant. For several years, it has been primarily used as a cordage crop and secondarily as a livestock feed. The potential for using kenaf seeds as a source of food-based products has not been fully exploited. Consumers are becoming more interested in naturally healthy plant-based food products. Kenaf seed, the future crop with a rich source of essential nutrients and an excellent source of phytocompounds, might serve suitable roles in the production of value-added plant-based foods. At present kenaf seed and its value-added components have not been effectively utilized for both their nutritional and functional properties as either ingredient or major constituent of food products. This review focuses on the possible food applications of kenaf seed and its value-added components based on their nutritional composition and functional properties available in literature, with the purpose of providing an overview on the possible food applications of this underutilized seed. The review focuses on a brief introduction on kenaf plant, nutritional function, lipids and proteins composition and food applications of the seed. The review elaborately discusses the seed in terms of; bioactive components, antioxidants enrichment of wheat bread, antimicrobial agents, as edible flour, as edible oil and a source of protein in food system. The review closes with discussion on other possible food applications of kenaf seed. The need for food scientists and technologists to exploit this natural agricultural product as a value-added food ingredient is of great significance and is emphasized.
\end{abstract}

Keyword: Composition; Food application; Functional properties; Kenaf seed; Potential 